\title{
Consumo de fibras e líquidos em mulheres participantes de uma universidade da terceira idade no Sul do Brasil
}

\author{
Renata Sandri", Juliana Rombaldi Bernardi", Josiane Siviero"**
}

\section{Resumo}

A ingestão de fibras alimentares e de líquidos vem há muito tempo despertando interesse de especialistas das áreas de nutrição e saúde em razão dos seus benefícios. O consumo de fibras concomitante à ingestão adequada de líquidos, especialmente a água, previne a constipação, favorece um melhor controle da glicemia e do colesterol e reduz o risco de algumas neoplasias. Este estudo observacional, transversal, retrospectivo, descritivo e analítico teve como objetivo avaliar o consumo de fibras e líquidos em mulheres participantes de uma universidade da terceira idade e comparar com recomendações nutricionais. Utilizando-se o banco de dados do projeto NUTENV, foram avaliadas 60 mulheres com registros completos de identificação, dados socioeconômicos e culturais, sintomatologia, antropometria e questões sobre as práticas/os consumo alimentares que envolveram a ingestão de água e fibras. A média etária foi de $64,3 \pm 8,0$ anos, regis- trando-se que $73,3 \%$ possuíam $\geq 60$ anos. Prevaleceram sobrepeso, obesidade e risco elevado de desenvolver doenças cardiovasculares, com base na medida da cintura. $\mathrm{O}$ IMC médio foi de $28,6 \mathrm{~kg} / \mathrm{m}^{2} \pm 6,0$, a ingestão média de líquidos foi de 1805+897 ml e de fibras, através do relato do R24h, foi de $22,3 \mathrm{~g} \pm 13,0$. A quantidade de fibras e líquidos para a maioria da amostra foi inadequada quando comparada às recomendações. Houve associação da idade com o total de água ingerida e com consumo de fibras insolúveis $(* p \leq 0,05)$. Como apontam os resultados encontrados no presente estudo, faz-se necessária a intensificação de medidas de educação nutricional e alimentar para a melhoria da ingestão desses nutrientes em adultos e idosos.

Palavras-chave: Idoso. Fibra na dieta. Ingestão de líquidos. Comportamento alimentar. Constipação intestinal.

* Acadêmica do curso de graduação em Nutrição da Universidade de Caxias do Sul. Endereço para correspondência: BR 116, Km 119, Pedras Brancas, Bairro Pedras Brancas, CEP: 95190-000, São Marcos - RS. Email: renatacsandri@hotmail.com.

** Nutricionista. Mestra em Ciências da Saúde pela Universidade Federal de Ciências da Saúde de Porto Alegre. Docente do curso de Nutrição da Universidade de Caxias do Sul e da Faculdade Cenecista de Bento Gonçalves.

**** Nutricionista. Doutora em Ciências da Saúde pela Pontifícia Universidade Católica do Rio Grande do Sul. Mestra em Gerontologia Biomédica pela Pontifícia Universidade Católica do Rio Grande do Sul. Docente do curso de Nutrição da Universidade de Caxias do Sul e da Faculdade Cenecista de Bento Gonçalves.

$\hookrightarrow$ doi:10.5335/rbceh.2012.020 


\section{Introdução}

O envelhecimento, pouco tempo atrás considerado um fenômeno, atualmente, faz parte da realidade da maioria das sociedades. (BRASIL, 2006a). Com o avanço no crescimento da população de adultos e idosos, considerando as projeções demográficas, tem-se uma preocupação com as demandas e necessidades de atenção à saúde, às questões socioeconômicas, culturais, biológicas, entre outras. Muñiz, Martínez e Cos Blanco (2004) mencionam que as alterações biológicas, psicológicas, sociais e as questões econômicas relacionadas a essas fases da vida podem afetar a alimentação ou a físiologia da nutrição, por meio de modificações no padrão alimentar e no estado nutricional.

A alimentação saudável é definida como uma condição alimentar que deve ser variada, balanceada e equilibrada em seus nutrientes. Considerando o universo dos grupos alimentares, merece destaque o grupo das frutas, por conter micronutrientes fundamentais para a prevenção e/ou o tratamento de diversas doenças crônicas. (SILVA; RECINE; QUEIROZ, 2002).

As fibras alimentares vêm há muito tempo despertando interesse de especialistas das áreas de nutrição e saúde em razão dos seus benefícios. (MATTOS; MARTINS, 2000). De acordo com Marchini et al. (1998), o consumo de fibras, associado a uma ingestão hídrica adequada, previne a constipação, favorece melhor controle da glicemia e do colesterol e reduz o risco de algumas neoplasias.

Alimentos ricos em fibras devem ser consumidos diariamente, porém, a maioria dos indivíduos os consome com menor frequência. As fibras, tanto solúveis quanto insolúveis, têm como seu maior alvo o trato gastrintestinal, servindo de substrato para a microbiota, ao atuar na velocidade de digestão e absorção dos nutrientes. (CUPPARI, 2005). A despeito de seus benefícios, Colli et al. (2002) afirmam que o excesso de fibras pode interferir na absorção de minerais como cálcio e zinco.

Associada ao consumo de fibra, recomenda-se a adequada ingestão de líquidos, entre os quais, a água, cuja ingestão é importante na saúde dos adultos e idosos, pois o próprio processo de envelhecimento, a atividade física, a medicação, a função renal, mecanismos homeostáticos e a redução da sensação de sede podem atuar como fatores contribuintes à constipação. (ARBONÉS et al., 2003).

Assim, este estudo teve como objetivo avaliar o consumo de fibras e líquidos em mulheres participantes de uma universidade da terceira idade no Sul do Brasil, comparando-o com recomendações nutricionais.

\section{Materiais e métodos}

Trata-se de um estudo observacional, transversal, retrospectivo, descritivo e analítico, utilizando-se o banco de dados do projeto NUTENV, intitulado "Alimentação e envelhecimento: estudo do perfil nutricional, antropométrico e a relação com fatores socioeconômicos e culturais de mulheres adultas e idosas participantes da Universidade da Terceira Idade da Universidade de Caxias do Sul" (UCS), aprovado pelo Comitê de 
Ética em Pesquisa (CEP) da UCS, sob o parecer n. 061/2009.

Nas entrevistas que deram origem ao banco de dados, as avaliadas iam sendo convidadas a participar do estudo. Em entrevista individual previamente agendada e com duração de 30 a 60 minutos, a pesquisa foi aplicada por nutricionista e/ou estudantes de nutrição treinadas para tal. As participantes eram entrevistadas, após assinarem, voluntariamente, o Termo de Consentimento Livre e Esclarecido (TCLE). Foram incluídas na amostra 60 mulheres com $\geq 50$ anos e registros completos.

Os dados de identificação, socioeconômicos e culturais compreenderam idade, estado civil, escolaridade e renda. As variáveis de sintomatologia foram avaliadas pela presença de constipação e frequência de evacuação. Para diagnosticar a primeira variável, o critério foi a resposta para a pergunta que investigava se havia ou não constipação. Em relação à frequência de evacuação, o critério utilizado foi: intestino/fezes (1) diariamente, (2) 2x semana, (3) 3x semana, (4) outro.

Nas variáveis antropométricas, foram registrados peso, estatura e circunferência da cintura (CC). Para a mensuração do peso, foi utilizada uma balança antropométrica Welmy ${ }^{\circledR}$, e estatura foi obtida utilizando estadiômetro acoplado à mesma balança. O Índice de Massa Corporal (IMC) foi calculado e classificado conforme a World Health Organization (WHO, 1997). A CC foi obtida circundando as mulheres com fita métrica inflexível, no ponto médio entre a última costela e a crista ilíaca, no momento da expiração. Os pontos de corte para CC foram da WHO (1997).
As variáveis sobre prática/consumo alimentar foram obtidas por meio de um questionário sobre as práticas de ingestão de líquidos e recordatório alimentar "habitual" de 24 horas (R24h).

Para avaliação da ingestão dietética de fibras e líquidos, foram utilizados os parâmetros do Institute of Medicine, Dietary Reference Intakes (DRIs, 2006). A recomendação de fibras para mulheres de 50-70 anos é $25 \mathrm{~g} /$ dia e para mulheres com idade $\geq 70$ anos, $21 \mathrm{~g} /$ dia; para líquidos, de $>19$ a 70 anos, a recomendação é 2,7 l/dia. Cabe considerar que, na determinação das DRIs, relaciona-se que todas as fontes de água podem contribuir para atender à necessidade total de líquidos (chás, cafés, sucos, água e umidade dos alimentos).

A ingestão de fibras e líquidos também foi avaliada com base nas recomendações do Guia Alimentar para a População Brasileira. (BRASIL, 2006b). Para fibras, a recomendação diária é $25 \mathrm{~g}$ e para líquidos, 21 de água (6-8 copos) ou $1 \mathrm{ml} / \mathrm{Kcal}$. Considerando um plano alimentar de $2.000 \mathrm{Kcal} /$ dia, recomenda-se a ingestão de $2.000 \mathrm{ml}$ de líquidos/água.

Após o cálculo do R24h, feito por meio do Software Dietwin Profissional ${ }^{\circledR}$ (vs.1995-2002), foram obtidas as quantidades totais de fibras, fibra solúvel e insolúvel, calorias, líquidos e água.

Os dados foram analisados com auxílio do pacote estatístico Statistical Package for the Social Sciences (SPSS $®$ ) vs. 18.0. As variáveis quantitativas foram descritas por média e desvio padrão (distribuição simétrica) ou mediana e amplitude interquartílica (distribuição assimétrica) acrescidos da amplitude de variação (valores mínimos e máximos). 
As variáveis qualitativas foram descritas por frequências absolutas e relativas. Para avaliar a associação entre os dados demográficos, antropométricos e calorias $24 \mathrm{~h}$ com a ingestão de líquidos e fibras, o teste da correlação de Spearman foi aplicado. Para comparar a ingestão de líquidos e fibras entre constipados e não constipados, foi utilizado o teste de Mann-Whitney. O nível de significância adotado foi de $5 \%(\mathrm{p} \leq 0,05)$.

\section{Resultados}

Foram avaliadas 60 mulheres adultas e idosas. Em relação à caracterização demográfica da amostra, verificou-se que a média etária foi $64,3 \pm 8,0$ anos ( mínima $=51$ anos e máxima $=86$ anos), $73,3 \%$ tendo idade $>60$ anos. A maioria, $56,7 \%$, era casada, seguida de $28,3 \%$ de viúvas, $11,7 \%$ de divorciadas e apenas $3,3 \%$ de solteiras. Analisando-se a escolaridade, verificou-se que $26,7 \%$ têm ensino médio completo; $23,3 \%$, superior completo; $20 \%$, fundamental incompleto; $13,3 \%$, fundamental completo; $8,3 \%$, en- sino médio incompleto; e 8,3\%, superior incompleto.

A renda média foi 1.516,00 $\pm 603(\mathrm{em}$ reais), sendo $R \$ 545,00$ a renda mínima e $R \$ 2.180,00$ a máxima, equivalente à média de 2,8 $\pm 1,1$ salários [mínimo = $1-$ máximo $=4$ ]. Somente $13,3 \%$ participantes ganhavam até um salário mínimo; a maioria, $48,3 \%$, ganhava $2-3$ salários; e 38,4\%, $\geq 4$ salários.

Ao avaliar o relato de constipação, constatou-se que apenas $26,7 \%$ das mulheres apresentaram esse sintoma, e a maioria, $78,3 \%$, relatou possuir frequência de evacuação diária. As demais relataram a seguinte frequência: $6,7 \%$, 3 vezes/semana; 3,3\%, 2 vezes/semana; e $11,7 \%$, outras.

Na Tabela 1, encontram-se a caracterização antropométrica e as calorias ingeridas pelo R24h. De acordo com a antropometria, o IMC foi de $28,6 \mathrm{~kg} /$ $\mathrm{m}^{2}+6,0$, sendo $71,7 \%$ classificadas em sobrepeso e obesidade. Ao verificar a classificação da CC, 71,7\% ficaram acima dos valores esperados.

Tabela 1 - Caracterização antropométrica e calorias ingeridas pelo recordatório de R24h.

\begin{tabular}{|c|c|}
\hline Variáveis & Amostra total $(n=60)$ \\
\hline IMC (kg/m2) - Média \pm DP [mínimo - máximo] & $28,6 \pm 6,0[18,1-46,0]$ \\
\hline \multicolumn{2}{|l|}{ Classificação do IMC - n(\%) } \\
\hline Baixo peso $\left(<18,5 \mathrm{~kg} / \mathrm{m}^{2}\right) /$ Eutrofia $\left(18,5-24,9 \mathrm{~kg} / \mathrm{m}^{2}\right)$ & $17(28,3)$ \\
\hline Sobrepeso $\left(25,0-29,9 \mathrm{~kg} / \mathrm{m}^{2}\right) /$ Obesidade $\left(\geq 30-34,9 \mathrm{~kg} / \mathrm{m}^{2}\right)$ & $43(71,7)$ \\
\hline Circunferência da cintura $(\mathrm{cm})-$ Média \pm DP [mínimo - máximo] & $88,5 \pm 13,3[63-132]$ \\
\hline \multicolumn{2}{|l|}{ Classificação da CC - n(\%) } \\
\hline Normal $(<80 \mathrm{~cm})$ & $17(28,3)$ \\
\hline Elevada $(\geq 80 \mathrm{~cm})$ & $43(71,7)$ \\
\hline Calorias (R24h) - Média \pm DP [mínimo - máximo] & $1519 \pm 492[711-2919]$ \\
\hline
\end{tabular}


A Tabela 2 permite visualizar a avaliação do consumo de líquidos. No que se refere à ingestão total de água pura relatada, somente uma participante afirmou consumir a quantidade recomendada pela DRIs. Tomando por base o Guia Alimentar para a População Brasileira, $18,3 \%$ das avaliadas revelaram consumir a quantidade total de água recomendada e 23,3\%, água suficiente, considerando o critério $\mathrm{ml} / \mathrm{Kcal}$, conforme o plano alimentar descrito. Em relação ao total de líquidos obtidos pelo R24h, apenas $10 \%$ os ingeriram adequadamente, segundo $o$ critério $\mathrm{ml} / \mathrm{Kcal}$, de acordo com o plano alimentar.

A média de ingestão total de outros líquidos foi $839 \pm 680 \mathrm{ml}$; destes, o maior consumo relatado foi de leite, seguido de chimarrão, sucos, chás e refrigerantes. A média de refrigerantes relatada foi baixa.

Relacionando a ingestão total de líquidos, ingestão média de $1805 \pm 897$ $\mathrm{ml}$, em comparação com as DRIs, apenas $13,3 \%$ atenderam às recomendações. Em relação ao guia alimentar, $35 \%$ os ingeriam dentro do preconizado e $55 \%$ atenderam à recomendação de ml de líquidos por calorias (Tabela 2). Ao transformar o relato de ingestão média de água e líquidos em copos de $250 \mathrm{ml}$, encontrou-se que as avaliadas ingeriam 3,8 copos/ água e 7,2 copos/líquidos. A mediana foi de 3 copos/água, variando entre 2-4 copos de água, e para os líquidos, 6,8 copos, variando entre 5-8,9 copos.

Tabela 2 - Avaliação do consumo de líquidos.

\begin{tabular}{|c|c|c|c|c|c|c|}
\hline Variáveis & $\begin{array}{l}\text { Média } \\
\pm \mathrm{DP}\end{array}$ & $\begin{array}{c}M d \\
(\mathrm{P} 25-\mathrm{P} 75)\end{array}$ & $\begin{array}{l}\text { Min. } \\
\text { - Máx. }\end{array}$ & $\begin{array}{c}\text { Ingestão } \\
\text { recomendada } \\
\text { DRls - } \\
2.700 \mathrm{ml} \\
\mathrm{n}(\%)\end{array}$ & $\begin{array}{c}\text { Ingestão } \\
\text { recomendada } \\
\mathrm{GA}-2.000 \mathrm{ml} \\
\mathrm{n}(\%)\end{array}$ & $\begin{array}{c}\text { Ingestão } \\
\text { recomendada } \\
\mathrm{GA}-1 \mathrm{ml} / \\
\text { calorias n (\%) }\end{array}$ \\
\hline $\begin{array}{l}\text { Total de água } \\
\text { relatada (ml) }\end{array}$ & $965 \pm 651$ & $750(500-1150)$ & $0-3000$ & $1(1,7)$ & $11(18,3)$ & $14(23,3)$ \\
\hline $\begin{array}{l}\text { Total de líquidos } \\
\text { R24h (ml) }\end{array}$ & $962 \pm 334$ & 985 (665-1165) & $403-1938$ & $0(0,0)$ & $0(0,0)$ & $6(10,0)$ \\
\hline $\begin{array}{l}\text { Total de outros } \\
\text { líquidos (ml) }\end{array}$ & $839 \pm 680$ & $725(362-1072)$ & $0-3500$ & - & - & - \\
\hline Sucos & $145 \pm 261$ & $0(0-250)$ & $0-1250$ & - & - & - \\
\hline Chás & $125 \pm 159$ & $0(0-150)$ & $0-500$ & - & - & - \\
\hline Refrigerante & $20,8 \pm 83,5$ & $0(0-0)$ & $0-500$ & - & - & - \\
\hline Chimarrão & $172 \pm 459$ & $0(0-112)$ & $0-3000$ & - & - & - \\
\hline Leite & $209 \pm 191$ & $150(0-300)$ & $0-1000$ & - & - & - \\
\hline $\begin{array}{l}\text { Total de água + } \\
\text { Total de outros } \\
\text { líquidos (ml) }\end{array}$ & $1805 \pm 897$ & $\begin{array}{l}1700(1250- \\
2237)\end{array}$ & $0-4720$ & $8(13,3)$ & $21(35,0)$ & $33(55,0)$ \\
\hline
\end{tabular}

$\mathrm{n}=$ número de mulheres avaliadas; $\mathrm{DP}=$ desvio padrão; $\mathrm{Md}=$ mediana; $\mathrm{P}=$ percentil $; \mathrm{ml}=$ mililitros; DRIs = dietary reference intakes; GA= Guia Alimentar para a população brasileira; $\mathrm{R} 24 \mathrm{~h}=$ recordatório de 24 horas. 
Na Tabela 3, que analisa o consumo de fibras pelo R24h, 28 idosas (46,7\%) as consomem na quantidade recomendada pelas DRIs e $21(35 \%)$ de acordo com o
Guia Alimentar. No que se refere ao tipo de fibra, verificou-se que houve maior consumo de fibras insolúveis.

Tabela 3 - Avaliação do consumo de fibras.

\begin{tabular}{|c|c|c|c|c|c|}
\hline Variáveis & Média \pm DP & $\begin{array}{c}M d \\
\text { (P25-P75) }\end{array}$ & $\begin{array}{l}\text { Min. } \\
\text { - Máx. }\end{array}$ & $\begin{array}{c}\text { Ingestão } \\
\text { recomendada DRIs - } \\
21 \mathrm{~g} / \mathrm{dia} \\
\mathrm{n}(\%)\end{array}$ & $\begin{array}{c}\text { Ingestão } \\
\text { recomendada } \\
\mathrm{GA}-25 \mathrm{~g} / \mathrm{dia} \\
\mathrm{n}(\%)\end{array}$ \\
\hline Fibras R24h & $22,3 \pm 13,0$ & $\begin{array}{c}20,5 \\
(12,1-29,0)\end{array}$ & $6,5-69,0$ & $28(46,7)$ & $21(35,0)$ \\
\hline Fibras solúveis & $4,5 \pm 3,7$ & $4,0(1,8-6,6)$ & $0,0-20,3$ & - & - \\
\hline Fibras insolúveis & $6,7 \pm 5,8$ & $4,8(2,7-10,6)$ & $0,0-28,7$ & - & - \\
\hline
\end{tabular}

$\mathrm{n}=$ número de mulheres avaliadas; $\mathrm{DP}=$ desvio padrão; $\mathrm{Md}=$ mediana $\mathrm{P}=$ percentil $;$ DRIs $=$ dietary reference intakes; GA= Guia Alimentar para a População Brasileira; R24h= recordatório de 24 horas.

Conforme Tabela 4, verificou-se que houve associação positiva estatisticamente significativa entre as calorias do R24h com o total de líquidos obtidos através do R24h, fibras do R24h e fibras insolúveis, ou seja, quanto maior o consumo de calorias no R24h, maior o total de líquidos ingerido pelo R24h, maior consumo de fibras e maior o consumo de fibras insolúveis. Também, houve associação inversa da idade com o total de água relatada e com o consumo de fibras insolúveis, ou seja, quanto maior a idade, menor o total de água relatada e menor o consumo de fibras insolúveis. Não houve associação significativa entre IMC, CC, renda e escolaridade com o total de líquidos e fibras ingeridos.

Tabela 4 - Associação entre líquidos e fibras ingeridos com dados demográficos, antropométricos e calorias, conforme coeficiente de correlação de Spearman.

\begin{tabular}{lcccccc}
\hline \multicolumn{1}{c}{ Variáveis } & IMC & CC & Calorias & Idade & Renda & Escolaridade \\
\hline Total de água relatada $(\mathrm{ml})$ & $-0,107$ & $-0,080$ & 0,015 & $-0,286^{*}$ & $-0,009$ & 0,186 \\
Total de líquidos do R24h $(\mathrm{ml})$ & $-0,056$ & 0,058 & $0,405^{* * *}$ & $-0,193$ & $-0,036$ & $-0,188$ \\
Total de outros líquidos $(\mathrm{ml})$ & 0,216 & 0,189 & $-0,073$ & $-0,049$ & 0,133 & 0,224 \\
$\begin{array}{l}\text { Total de água + Total de líquidos } \\
(\mathrm{ml})\end{array}$ & 0,139 & 0,122 & $-0,049$ & $-0,215$ & 0,062 & 0,214 \\
Fibras R24h & $-0,093$ & $-0,123$ & $0,329^{* *}$ & $-0,042$ & 0,155 & 0,008 \\
Fibras solúveis & $-0,106$ & $-0,056$ & $0,295^{*}$ & $-0,181$ & 0,197 & 0,043 \\
Fibras insolúveis & $-0,139$ & $-0,123$ & $0,283^{*}$ & $-0,260^{*}$ & 0,160 & 0,066 \\
\hline
\end{tabular}

${ }^{*} \mathrm{p} \leq 0,05 ;{ }^{* *} \mathrm{p} \leq 0,01 ;{ }^{* * * *} \mathrm{p} \leq 0,001 ; \mathrm{ml}=$ mililitros; $\mathrm{R} 24 \mathrm{~h}=$ recordatório de 24 horas; $\mathrm{IMC}=$ índice de massa corporal; $\mathrm{CC}=$ circunferência da cintura. 
A Tabela 5 apresenta a avaliação da ingestão de líquidos e fibras conforme presença ou não de constipação.
Verificou-se que não houve diferença significativa na ingestão de líquidos e fibras dos constipados e não constipados.

Tabela 5 - Avaliação da ingestão de líquidos e fibras conforme constipação.

\begin{tabular}{lccc}
\hline \multicolumn{1}{c}{ Variáveis } & $\begin{array}{c}\text { Constipados } \\
(\mathrm{n}=16)\end{array}$ & $\begin{array}{c}\text { Não constipados } \\
(\mathrm{n}=44)\end{array}$ & $\mathrm{p}^{*}$ \\
& $\mathrm{Md}(\mathrm{P} 25-\mathrm{P} 75)$ & $\mathrm{Md}(\mathrm{P} 25-\mathrm{P} 75)$ & \\
\hline Total de água relatada $(\mathrm{ml})$ & $1000(500-2000)$ & $750(500-1000)$ & 0,610 \\
Total de líquidos R24h $(\mathrm{ml})$ & $1063(858-1119)$ & $862(634-1166)$ & 0,349 \\
Total de outros líquidos $(\mathrm{ml})$ & $525(300-993)$ & $800(450-1110)$ & 0,353 \\
Total de água + Total de outros líquidos $(\mathrm{ml})$ & $1925(850-2375)$ & $1525(1250-2175)$ & 0,732 \\
Fibras R24h & $20,1(11,1-25,2)$ & $20,5(12,1-29,4)$ & 0,547 \\
Fibras solúveis & $4,0(2,1-6,4)$ & $4,2(1,7-6,7)$ & 0,422 \\
Fibras insolúveis & $4,6(2,5-7,5)$ & $4,9(2,8-10,7)$ & 0,980 \\
\hline
\end{tabular}

* teste de Mann-Whitney; $\mathrm{n}=$ número de mulheres avaliadas; $\mathrm{ml}=$ mililitros; $\mathrm{R} 24 \mathrm{~h}=$ recordatório de 24 horas; $\mathrm{Md}=$ mediana; $\mathrm{P}=$ percentil.

\section{Discussão}

A média de idade das avaliadas foi $64,3 \pm 8,0$ anos, tendo a maior parte delas $\geq 60$ anos. Inúmeros grupos de convivência, instituições de longa permanência, universidades para adultos e idosos contam, em sua maioria, com a participação de mulheres. (CERVATO et al., 2005). As participantes deste estudo fazem parte da UNTI, que tem ampla adesão de mulheres. (CORTELETTI; CASARA, 2007). Esse predomínio do gênero feminino é corroborado pelos dados do IBGE (1999), segundo os quais a população feminina encontra-se em maior número e teve crescimento intenso, com média de 118 mulheres para cada 100 homens na faixa etária de 65-69 anos e de 141 para cada 100 no grupo de 80 anos ou mais.
Constatou-se que a maioria das idosas apresentava bom nível socioeconômico. De acordo com o IBGE (1999), as condições socioeconômicas desempenham papel fundamental sobre a qualidade de vida na velhice. Renda suficiente para atendimento das necessidades básicas, bom nível de escolaridade, relações familiares adequadas, boas condições de sociabilidade e atividade produtiva podem contribuir para maior autonomia do idoso. Além disso, talvez por apresentarem maior escolaridade, reconhecem a necessidade do autocuidado, que envolve melhoria na saúde e prática de atividades de lazer, buscando atividades sociais e culturais. (CERVATO et al., 2005). No caso da amostra, a opção por frequentar uma universidade da terceira idade pode refletir esse aspecto. 
Observou-se que mais da metade das mulheres avaliadas, 71,7\%, encontrava-se com sobrepeso e obesidade, semelhantemente ao encontrado por Rauen, Moreira e Calvo (2006) em estudo com idosos institucionalizados, com maior prevalência de excesso de peso $(41 \%) \mathrm{e}$ percentuais menores para eutrofia e magreza, em $45 \%$ e $14 \%$, respectivamente.

Tavares e Anjos (1999), com base em uma análise do perfil antropométrico da população idosa brasileira feita com 4.277 idosos (7,2\% da amostra total), apontaram prevalência de obesidade de $5,7 \%$ nos homens e de $18,2 \%$ nas mulheres, relacionando o aumento dessa prevalência nos idosos que vivem na região Sul do Brasil. Esse estudo, apesar de ter avaliado ambos os gêneros, ressalta a presença de percentual maior de mulheres obesas do que de homens obesos. Silveira, Lopes e Caialfa (2007) em estudo multicêntrico com idosos brasileiros, acompanhados em ambulatórios, identificaram que 36\% das mulheres estavam com IMC $>27,3$ $\mathrm{kg} / \mathrm{m}^{2}$. Os autores relacionaram que a prevalência de obesidade nas mulheres poderia ser atribuída ao maior acúmulo de gordura visceral.

Fiore et al. (2006), ao avaliarem a $\mathrm{CC}$, encontraram elevada prevalência de risco para doenças cardiovasculares e para os distúrbios metabólicos, com destaque para o sexo feminino. Trata-se de resultados semelhantes ao encontrado neste estudo, em que a maioria das mulheres obteve risco elevado para desenvolver doenças cardiovasculares.

A prática da ingestão de líquidos é de evidente importância, e nesse sentido a água se destaca, pois desempenha papel fundamental na regulação de muitas funções vitais do organismo, incluindo a regulação da temperatura, a participação no transporte de nutrientes, na eliminação de substâncias tóxicas, bem como nos processos digestivo, respiratório, cardiovascular e renal. (BRASIL, 2006b).

Verificou-se baixa ingestão de líquidos em relação às recomendações das DRIs e do Guia Alimentar. Resultado um pouco superior foi encontrado quando se avaliou a quantidade total de líquidos com base no critério da ingestão de $\mathrm{ml}$ por calorias. Encontrou-se resultado semelhante em um estudo realizado em São Paulo, apontando o baixo consumo de água, segundo o qual $22,5 \%$ dos idosos tinham hábito de ingerir apenas 1 copo de água/dia, $45 \%$ consumiam entre $2-3$ copos, 17,5\% consumiam de $4-5,10 \%$ ingeriam de 6-7 e 5\% dos idosos consumiam $\geq 8$ copos. (SILVA; CARLUCIO, 2007). No presente estudo, a ingestão média foi de 3,8 copos de água e de 7,2 copos de líquidos, com mediana de 3 copos, variando entre 2-4 copos de água $\mathrm{e}$ 6,8 copos de líquidos (5-8,9 copos).

Estudo realizado em Erechim - RS, avaliando a ingestão de líquidos e água em adultos/idosos, encontrou consumo médio de 5,8 copos de água para adultos e 5,7 copos para idosos. O consumo médio de líquidos foi de 9 copos para adultos e 9,1 copos para idosos. (CARVALHO; ZANARDO, 2010). Apesar de não se ter analisado separadamente a quantidade de ingestão de água e líquidos para adultos e idosos, observou-se que a ingestão média de água e líquidos é menor quando 
comparada aos achados do estudo realizado em Erechim.

Segundo Waitzberg (2004), a proporção de água no organismo varia de acordo com a idade. Os idosos, além de possuírem menor quantidade de água, cerca de 40 a $50 \%$ do peso corpóreo, são mais vulneráveis à sua perda do que os jovens. Eles tendem a perder soluções isotônicas para o exterior e ingerem menor quantidade de líquidos, o que exige que o organismo elimine uma quantidade maior de eletrólitos, com relação à quantidade de água eliminada. Seus rins e glândulas endócrinas são menos capazes de produzir esses ajustes finos de retenção de água. Somando-se a esse fato, a arterosclerose pode diminuir a sensação de sede, o que contribui, ainda, para a não reposição de líquido perdido.

$\mathrm{O}$ maior consumo de outros líquidos relatado entre as mulheres neste estudo foi de leite. É possível que elas se mostrem mais sensibilizadas sobre os benefícios desse produto devido a uma maior preocupação com a saúde, no que se refere à prevenção de doenças ósseas como a osteoporose, e também pela realização de exames preventivos para esse fim. Kurnik et al. (2010) avaliaram o consumo de leite e derivados em idosas fisicamente ativas, observando que a maioria delas consumia ao menos uma porção diária do produto, semelhantemente ao apresentado pelas avaliadas neste estudo.

Ainda sobre os outros líquidos, verificou-se que o consumo de refrigerante foi baixo, o que é um dado positivo, tendo em vista que estes são ricos em carboidrato simples, os quais podem ter relação direta com sobrepeso/obesidade. Pereira et al. (2009), em estudo com mulheres adultas, verificaram que houve redução no consumo de bebidas com adição de açúcar, incluindo-se os refrigerantes.

Ao verificar o consumo de fibras totais, nossos achados mostraram que a ingestão foi inadequada, pois menos da metade da amostra (46,7\%) atingiu a quantidade recomendada pelas DRIs e apenas $35 \%$ estiveram de acordo com o Guia Alimentar. Ao comparar esses resultados com os de outros estudos, observou-se, na Inglaterra, que somente 5 a $25 \%$ de sua população atende às recomendações de consumo de fibras, que é de 25-30 g diárias. (WHO, 2002). No Brasil, o número de pesquisas relacionadas ao consumo de fibras tem crescido, dada a importância da sua ingestão para a prevenção de doenças, como, por exemplo, diverticulite do cólon, câncer colorretal e diabetes mellitus. Porém, dados sobre a ingestão de fibras em nível nacional são escassos.

Mattos e Martins (2000), estudando uma amostra da população do município de Cotia - SP composta por 559 indivíduos com $\geq 20$ anos, relataram baixo consumo de fibras. Resultados similares foram encontrados por Federmann (1994), que investigou o consumo de fibras em indivíduos diabéticos, verificando que $92 \%$ deles as ingeriram abaixo das recomendações.

No presente estudo, observou-se que as mulheres estão consumindo maiores quantidades de fibras insolúveis, encontradas nos produtos integrais, nas frutas, hortaliças e raízes. (ROCHA et al., 1999). As fibras apresentam efeitos 
benéficos ao organismo. Enquanto as insolúveis são importantes por facilitar a ação peristáltica do intestino, as solúveis têm a propriedade de se ligarem à água, formando um gel que reduz a absorção de lipídios e açúcares, tornando-se substrato para a formação de rica flora bacteriana. Além de serem eficazes na regulação dos níveis plasmáticos de glicose, colesterol e triglicerídeos, as fibras constituem fator preventivo de certas enfermidades crônicas. (GONÇALVES, 2007).

Nesta pesquisa, verificou-se que, quanto maior a idade das mulheres, menor o total de água relatada e menor o consumo de fibras insolúveis. Isso se explica porque, com o avançar da idade, os idosos apresentam inúmeras mudanças no organismo, como a diminuição da percepção de sede, apetite, paladar e olfato, o que pode tornar a comida menos atrativa. (REIS, 2001). De acordo com Castro e Amâncio (2000), o uso de próteses dentárias é outro fator que pode afetar os idosos devido a uma mastigação menos eficiente, levando a um menor consumo de carnes, frutas e vegetais frescos e à preferência por alimentos de fácil mastigação.

A maioria das mulheres relatou não ser constipada, mesmo com a ingestão de fibras e de líquidos abaixo das recomendações. Também não houve diferenças significativas na ingestão de líquidos e fibras entre constipados e não constipados. Esse dado sugere que o tipo de fibra - nesse caso, a maior ingestão foi das insolúveis - pode ter influenciado esse resultado, além de outros fatores.
Por fim, cabe ponderar que as avaliadas podem ter subestimado os relatos tanto da ingestão de líquidos como do consumo de fibras.

\section{Conclusão}

As avaliadas, em sua maioria, eram mulheres idosas com bom nível de escolaridade e renda mensal. Prevaleceram o sobrepeso e a obesidade, além do risco elevado para desenvolver doenças cardiovasculares. Quanto à ingestão de fibras e líquidos, para a maioria da amostra ela foi inadequada quando comparada às recomendações. O consumo inadequado desses nutrientes, principalmente por parte da população idosa, pode acarretar diversos problemas de saúde. Por isso, é fundamental a adequação das quantidades de ingestão tanto de fibra como de líquidos, pois estes exercem inúmeras funções no organismo.

Portanto, os resultados encontrados no presente estudo apontam para a necessidade de intensificação de medidas de educação nutricional para a melhoria da ingestão desses nutrientes entre as mulheres. Também, é importante ressaltar que outros estudos devem ser implementados, investigando outros aspectos relacionados aos alimentos fontes de fibras e à ingestão de líquidos nessa mesma população. 
Consumption of fibers and liquids in women taking part in a university of the third age in Southern Brazil

\section{Abstract}

The intake of dietary fiber and liquids has long attracted interest from experts in the areas of nutrition and health due to its benefits. The concomitant intake of fiber adequate intake of fluids, especially water, prevents constipation, promotes a better control of blood glucose and cholesterol and reduces the risk of some cancers. This study aimed to evaluate the consumption of fiber and fluids in women participating in a university of the third age and compare it with nutritional recommendations. It is a cross-sectional study, retrospective analysis with descriptive and analytical, using the database project NUTENV. We evaluated 60 women with complete records of identification, socio economic and cultural symptoms, anthropometry and questions about the practices/food consumption involving the ingestion of water and fiber. The mean age was $64,3 \pm 8,0$ years, $73,3 \%$ were $\geq 60$ years. Prevailed overweight, obesity and increased risk of developing cardiovascular disease by measuring the waist. The mean IMC was $28,6 \mathrm{~kg} / \mathrm{m}^{2} \pm 6,0$. The average intake of liquids was $1805 \pm$ $897 \mathrm{ml}$ and fiber through the story of 24hour recall was $22,3 \pm 13,0 \mathrm{~g}$. The amount of fiber and liquids for most of the sample was inadequate when compared to the recommendations. There was an association of age with the total water consumed and intake of insoluble fiber $\left({ }^{*} p \leq 0,05\right)$. Based on the results of this study, it is necessary to intensify measures for food and nutrition education to improve the intake of these nutrients in adults and elderly.

Keywords: Elderly. Fiber in the diet. Liquids intake. Feeding behavior. Intestinal constipation.

\section{Agradecimentos}

Às participantes da Universidade da Terceira Idade de Caxias do Sul - RS e à Isabel M. Toni, responsável pela UNTI, por ter concordado com o estudo e por tê-lo apoiado.

\section{Referências}

ARBONÉS, G. et al. Nutrición y recomendaciones dietéticas para personas mayores. Grupo de trajo "Salud pública" de la Sociedad Española de Nutrición (SEN). Nutrição Hospitalar, Madrid, v. 18, n. 3, p. 109-137, 2003. BRASIL. Ministério da Saúde. Portaria 687 / GM, de 30 de março de 2006. Política Nacional de Promoção da Saúde, 2006a.

- Secretaria de Atenção à saúde. Coordenação-Geral da Política de Alimentação e Nutrição. Guia Alimentar para a população brasileira: promovendo a alimentação saudável/Ministério da Saúde, 2006b.

CARVALHO, A. P. I.; ZANARDO, V. P. S. Consumo de água e outros líquidos em adultos e idosos residentes no município de Erechim - Rio Grande do Sul. Perspectiva, Erechim, v. 34 , n. 125 , p. 117-126, mar. 2010.

CASTRO, A. G. P.; AMÂNCIO, O. M. S. Nutrição e fisiologia dos dentes. Revista de Sociedade Brasileira de Alimentação e Nutrição, São Paulo, v. 19, n. 20, p. 87-104, 2000.

CERVATO, A. M. et al. Educação nutricional para adultos e idosos: uma experiência positiva em universidade aberta para a terceira idade. Revista de Nutrição, Campinas, v. 18, n. 1, p. 41-52, jan./fev. 2005.

COLLI, C. et al. Alimentos funcionais. In: CUPPARI, L. (Org.). Guias de medicina ambulatorial e hospitalar: nutrição clínica no adulto. São Paulo: Manole, 2002. p. 55-70. 
CORTELETTI, I. A.; CASARA, M. B. Projeto pedagógico: Universidade da Terceira Idade - UNTI. Caxias do Sul: Educs, 2007. p. 7-25. CUPARRI, L. Guia de medicina ambulatorial e hospitalar: nutrição clínica no adulto. 2. ed. São Paulo: Manole, 2005.

FEDERMANN, M. Conhecimentos e práticas alimentares de indivíduos diabéticos não insulinodependentes. 1994. 155 f. Dissertação (Mestrado em Nutrição) - Faculdade de Saúde Pública, Universidade de São Paulo, São Paulo, 1994.

FIORI, E. G. et al. Perfil nutricional de idosos. Revista da Ciência Médica, Campinas, v. 15, n. 5, p. 369-377, set./out. 2006.

GONÇALVES, M. C. R. Fibras dietéticas solúveis e suas funções nas dislipidemias. Revista Brasileira de Nutrição Clínica, São Paulo, v. 22, n. 2, p. 167-173, 2007.

INSTITUTO BRASILEIRO DE GEOGRAFIA E ESTATÍSTICA (IBGE). Ministério do Planejamento, Orçamento e Gestão. Pesquisa sobre padrões de vida 1996-1997. 2. ed. Rio de Janeiro: IBGE, 1999.

INSTITUTE OF MEDICINE. Dietary reference intakes: the essencial guide to nutriente requirements. Washington: National Academy Press, 2006.

KURNIK, K. F. et al. Estudo do consumo de leite e derivados por idosas praticantes de atividade física em uma academia no município de São Paulo. Revista Digital E F Deportes, Buenos Aires, v. 15, n. 151, 2010.

MARCHINI, J. S.; FERRIOLI, E.; MORIGUTI, J. C. Suporte nutricional no paciente idoso: definição, diagnóstico, avaliação e intervenção. Medicina, Ribeirão Preto, v. 31, n. 1, p. 54-61, jan./mar. 1998.

MATTOS, L. L.; MARTINS, I. S. Consumo de fibras alimentares em população adulta. Revista de Saúde Pública, São Paulo, v. 34, n. 1, p. 50-55, fev. 2000.

MUÑIZ, C. M.; MARTÍNEZ, C. V.; COS BLANCO, A. I. Alimentación em El anciano sano. In: CANDELA, C. G. (Org.). Manual de recomendaciones nutricionales em pacientes geriátricos. Barcelona: Novartis, 2004. p. 99-108.

PEREIRA, R. A. et al. Mudanças no consumo alimentar de mulheres do município do Rio de Janeiro, Brasil, 1995-2005. Caderno de Saúde Pública, Rio de Janeiro, v. 25, n. 11, p. 2419-2432, nov. 2009.

RAUEN, M. S.; MOREIRA, E. A.; CALVO, M. C. Oral condition and its relationship to nutritional status in the institutionalized elderly population. Journal American Dietetic Association, Chicago, v. 106, n. 7, p. 1112-1114, jul. 2006.

REIS, N. T. Problemas relacionados à alimentação e qualidade de vida do idoso. Revista Nutrição em Pauta, São Paulo, v. 9, n. 48, p. 17-22, 2001.

ROCHA, R. P. et al. Consumo de fibras por adultos: estudo preliminar. Revista do Instituto Materno Infantil de Pernambuco, Recife, v. 13, n. 2, p. 138-143, 1999.

SILVA, D. O.; RECINE, E. G. I. G.; QUEIROZ, E. F. O. Concepções de profissionais de saúde da atenção básica sobre a alimentação saudável no Distrito Federal, Brasil. Caderno de Saúde Pública, Rio de Janeiro, v. 18, n. 5, p. 1367-1377, set./out. 2002.

SILVA, G. V.; CARLUCIO, B. F. Avaliação do estado nutricional de idosos residentes em um asilo na zona norte do estado de São Paulo, 2007. Disponível em: <http://boasaude.uol. com.br/realce/showdoc.cfm?libdocid=16185\& ReturnCatID=187>. Acesso em: 10 maio 2011.

SILVEIRA, E. A.; LOPES, A. C. S.; CAIALFA, W. T. Avaliação do estado nutricional de idosos. In: KAC, G., SICHIERI, R., GIGANTE, D. P. (Org.). Epidemiologia nutricional. Rio de Janeiro: Atheneu, 2007. p. 105-125.

TAVARES, E. L.; ANJOS, L. A. Perfil antropométrico da população idosa brasileira: resultados da Pesquisa Nacional sobre Saúde e Nutrição (PNSN). Caderno Saúde Pública, Rio de Janeiro, v. 15, n. 4, p. 759-768, 1999. 
WAITZBERG, D. L. Nutrição oral, enteral e parenteral na prática clínica. 3. ed. São Paulo: Atheneu, 2004. v. 1.

WORLD HEALTH ORGANIZATION (WHO). Obesity: preventing and managing the global epidemic. Geneva: Switzerland, 1999.

FAO. Diet, nutrition and the prevention of chronic diseases: report of a joint expert consultation. Geneva: Switzerland, 2002. 